\title{
PANDEMIA, SALUD MENTAL Y DESEMPEÑO ACADÉMICO DE LOS Y LAS ESTUDIANTES DE LA UNICAMACHO EN 2020
}

\section{PANDEMIC, MENTAL HEALTH AND ACADEMIC PERFORMANCE OF UNICAMACHO STUDENTS IN 2020}

\author{
Jimmy Ramírez Bejarano, Ivonne Melisa Lemos Carabalí, Johana Andrea Moncayo Eraso y Santiago Martínez \\ Bolaños. \\ Grupo de Investigación ANUDAMIENTOS \\ Institución universitaria Antonio José Camacho \\ Recibido: 30/03/2021 Aprobado: 24/05/2021 \\ Cómo citar este artículo: \\ Ramírez-Bejarano, J. A., Lemos-Carabalí, I.M., Martínez-Bolaños, S. y Moncayo-Erazo, J. A.(2021). Pandemia, salud \\ mental y desempeño académico de los y las estudiantes de la Unicamacho en 2020. Revista Sapientía, 13(25), 58 - 67.
}

\section{RESUMEN}

Esta investigación tuvo como objetivo caracterizar los efectos que la pandemia COVID-19 ha generado en la salud mental y el desempeño académico de los y las estudiantes de la Unicamacho. Es una investigación de tipo explicativo-comprensivo de corte trasversal, donde se aplicó una mirada de complementación cualitativa y cuantitativa. Este ejercicio se realizó como parte del curso Sistematización de Experiencias con estudiantes de sexto semestre de Trabajo social 2020-2. El tema se aborda desde la perspectiva actual de la psicología de la salud, que señala que el factor biológico, psicológico y los factores sociales tienen un papel fundamental en el contexto de una enfermedad o discapacidad. En ese sentido, la salud mental implica la idea de salud biopsicosocial. Se concluye que la pandemia ha sido un evento estresante altamente traumático que ha afectado la salud mental de gran parte de las y los estudiantes de la Unicamacho y afectó negativamente su rendimiento académico.

\section{PALABRAS CLAVE}

Salud mental, desempeño académico, pandemia.

\section{ABSTRACT}

This research aimed to characterize the effects that the COVID-19 pandemic has generated on the mental health and academic performance of Unicamacho students. It is an explanatory-comprehensive type of research, where a complementary look was applied that allowed the use of qualitative and quantitative approaches. The subject is approached from the current perspective of health psychology that indicates that the biological, psychological and social factors have a fundamental role in the context of a disease or disability in that sense, mental health implies the idea of biopsychosocial health. It is concluded that the pandemic has been a highly traumatic stressful event that has affected the mental health of a large part of the students, which negatively affected their academic performance.

\section{KEYWORDS}

Mental health, academic performance, pandemic. 


\section{INTRODUCCIÓN}

La pandemia del SARS-CoV-2 (COVID-19) afectó la vida de las personas alrededor del mundo; el aislamiento y demás medidas de distanciamiento social generaron efectos en lo social, lo económico, lo familiar y lo psicológico aún impredecibles. Para la población en general cambiaron de un momento a otro todas las rutinas diarias, familiares, de estudio, de trabajo, de entretenimiento y relacionamiento social.

Además de los efectos en la salud y en la economía, se ha generado un deterioro de la salud mental de las personas. Se generaron cuadros de estrés, ansiedad y pánico debido al distanciamiento y aislamiento social, a raíz de los conflictos ocasionados por las nuevas rutinas de estudio, de trabajo, económicas, familiares y sociales.

La pandemia afectó el sector de la educación, el cual no estaba preparado para un evento de estas dimensiones. La pandemia género que todas las instituciones educativas a todo nivel, básica y superior, cerrarán sus puertas y dejarán de operar presencialmente afectando a millones de estudiantes en nuestro país y en el mundo.

Estos cierres para contener la pandemia llevaron a una respuesta rápida que contempló la solución de la educación a distancia para poder dar continuidad a los procesos que se venían dando. Estas medidas, en muchos casos improvisadas, han enfrentado múltiples obstáculos a nivel tecnológico, pedagógico, económico y psicosocial.

El impacto más fuerte que ha tenido el cierre de las instituciones educativas y el inicio de la educación a distancia ha repercutido sobre todo en la calidad de la educación. De un momento a otro se cambiaron las metodologías para prestar servicios educativos y esto fue de gran impacto para estudiantes y profesores a nivel pedagógico y psicológico emocional.
La educación a distancia ha sido una solución de emergencia que ha generado resultados negativos en términos de calidad en el aprendizaje, pues los estudiantes no cuentan con los equipos, ni la tecnología y, mucho menos, con la conectividad a internet necesaria para cursar procesos educativos con calidad. La pandemia ha sacado a la luz las grandes y graves situaciones de desigualdad en las que se encuentran la población de estudiantil.

Igualmente, el cierre de las universidades y el inicio de la educación a distancia tomó por sorpresa a los docentes, quienes se vieron confrontados y fueron obligados a usar el recurso de la tecnología para dictar sus cursos sin tener la preparación suficiente, lo que llevó a los profesores a seguir dictando sus cursos por Internet, pero como si aún estuvieran en el aula, es decir, ni cambiaron sus currículos ni cambiaron sus metodologías.

Esta situación ha generado tanto en profesores como estudiantes una sensación de sentirse sobrepasados, asfixiados, abrumados, con altos niveles de ansiedad y estrés por la obligatoriedad de manipular tanta información a través de estas plataformas virtuales, más aún si se suman todas las limitaciones en conectividad y la falta de competencias para operar todo este tipo de plataformas de mediación tecnológica.

Otro aspecto importante que se debe resaltar es que tanto profesores y sobre todo los y las estudiantes han tenido que acomodar su vida cotidiana para adecuarse a la situación de aislamiento social. Muchos de los estudiantes que provienen de territorios y zonas rurales alejadas de la ciudad y que vivían en ella en razón de sus estudios, por causa de la pandemia han vuelto a sus lugares de origen haciendo inevitable el alejamiento social y la pérdida de las relaciones sociales y de amistad.

El aislamiento, las nuevas rutinas y la lógica de la educación a distancia están teniendo efectos fuertes en términos de equilibrio socioemocional y salud mental 
para los y las estudiantes. Por tanto, es imperativo que las instituciones deban atender, no solo las necesidades pedagógicas, sino además las necesidades socioemocionales de las y los estudiantes.

Todas las razones anteriores suscitaron el interés de conocer las afectaciones de la pandemia a nivel de salud mental y desempeño académico de las y los estudiantes de la institución universitaria Antonio José Camacho.

El presente estudio exploratorio nos proporcionará elementos empíricos que permitan proponer alternativas de atención a las y los estudiantes aportando a una formación efectiva que garantice su adecuada profesionalización en estos difíciles tiempos.

\section{SALUD MENTAL $Y$ AFECTACIONES EN EL DESEMPEÑO ACADÉMICO DE LAS Y LOS ESTUDIANTES}

Para hablar de salud mental es preciso primero reconocer que hay múltiples miradas y concepciones de salud mental según los diversos ámbitos y disciplinas en que se halla este concepto. Ramos (2015) reúne toda esta diversidad en dos modelos explicativos o paradigmas para entender la salud mental: el modelo biologicista, que asocia la salud a la ausencia de enfermedad, centrándose en la enfermedad y sus causas biológicas (fisicoquímicas) en el individuo solo y el modelo biopsicosocial, que plantea que lo biológico, lo psicológico y lo social son factores determinantes del bienestar en los procesos de salud-enfermedad. Es decir, que la vida cotidiana, las interacciones sociales, las experiencias vividas condicionan el bienestar físico, emocional y social de las personas.

Para la OMS, la salud mental es un estado de bienestar completo, físico, mental y social, y no meramente la ausencia de afecciones o enfermedad. Según Carrazana (2002), la salud mental es el estado que permite el desarrollo óptimo de cada individuo en el orden físico, intelectual y afectivo, en la medida en que es compatible con el desarrollo de otros individuos (p.10).

La salud mental como la capacidad de manejar las emociones y lograr vencer los obstáculos utilizando herramientas de control y manejo de las situaciones de la vida cotidiana (Zubiría, 2007) les permite a los seres humanos afrontar las presiones, realizar más eficazmente sus obligaciones y llevar mejores relaciones con las personas de su entorno, así como tener altos niveles de satisfacción.

Para el Ministerio de Salud (2011), un problema de salud mental afecta la forma cómo una persona piensa, se siente, se comporta y se relaciona con los demás. La salud mental está relacionada con algunas de nuestras capacidades básicas como pensar, relacionarnos, encontrar significado a la vida, capacidad para enfrentar cambios y manejar las crisis. Para la OMS (2006) síntomas frecuentes de los trastornos mentales son los sentimientos de tristeza, miedo, soledad, ansiedad y culpa.

Según Mebarak, De Castro, Salamanca, \& Quintero (2009), se considera a un individuo con buena salud mental si muestra la suficiente capacidad para relacionarse con su entorno de una manera flexible, productiva y adaptativa, y si sus percepciones de sí mismo y del entorno son constructivas y promotoras de equilibrio propio (p.89).

Ahora, la pandemia SARS-Cov2 (COVID-19) afectó la salud mental de las personas. Un estudio realizado por Huarcaya (2020) realiza una revisión narrativa de todos los artículos publicados desde el 1 de diciembre del 2019 hasta el 2 de marzo del 2020, relacionados directamente con los efectos psiquiátricos, psicológicos y de salud mental de la COVID-19, donde se demostró que desde la etapa inicial de la pandemia hubo un aumento de emociones negativas (ansiedad, depresión e indignación) y una disminución de emociones positivas (felicidad y satisfacción); por lo cual ha sido común la presencia de ansiedad, depresión y reacción al estrés en la población general. 
En el área de la educación encontramos que, según la OMS (2011), los problemas que está generando la pandemia en las y los estudiantes son deficiencia de atención, hiperactividad, ansiedad, depresiones, desórdenes en la alimentación y el sueño que dificultan el desempeño académico ( $p, 21)$.

Una investigación llevada a cabo por Cobo, Vega \& García (2020), cuyo propósito fue aportar algunas consideraciones institucionales sobre la salud mental en estudiantes universitarios durante la pandemia por COVID-19, a través de un diseño documental encontró que se ha presentado mayor incremento en síntomas depresivos, estrés y ansiedad. Además, identificó sentimientos de miedo extremo, dificultades en la duración del sueño e ideación suicida. Por otro lado, en Chile, Martínez (2020) realizó un estudio con el fin de identificar y analizar publicaciones cuya temática abordara la salud mental en confinamiento de estudiantes chilenos durante el año 2020, en la cual se concluyó que hubo un incremento en el estrés familiar, inseguridad financiera e incertidumbre, ya que la pandemia COVID-19 amenazó y afectó no solamente la salud física y mental, sino también la economía, intensificando aún más los trastornos de estrés, ansiedad, depresión y en general la sensación de temor a la vida o lo que se está viviendo. Por otro lado, llegó a la conclusión de que en estudiantes de educación superior existe mayor riesgo de que presenten problemas de salud mental originados por la pandemia; además, a nivel general se presentó una reacción negativa a la modalidad virtual que se adoptó para dar continuidad a la educación en la mayoría de las instituciones.

Ribot, Chang \& González (2020), en la investigación "Efectos de la COVID-19 en la salud mental de la población", sostienen que los efectos psicosociales tienen mayor impacto en poblaciones en condiciones de vulnerabilidad, dado que no cuentan con recursos necesarios para afrontar la situación y su acceso a los servicios sociales y de salud es limitado. Estos autores finalmente concluyen que la ansiedad, la preocupación y el miedo son los sentimientos que se experimentan con mayor frecuencia; advirtiendo, en primer lugar, la imperiosa necesidad de fortalecer la resiliencia, el crecimiento personal y las relaciones familiares, y, en segundo lugar, brindar especial atención a poblaciones vulnerables.

Cobo, Vega, \& García (2020) en su investigación sobre la salud mental en estudiantes universitarios expuestos al COVID-19, describen que situaciones económicas, así como los retrasos en las actividades académicas fueron identificados como factores de riesgo para el desarrollo de la ansiedad en estudiantes. Refiere que los síntomas que se han identificado en mayor medida han sido el incremento de los síntomas depresivos, estrés y ansiedad, estrés postraumático, sentimientos de miedo extremos, dificultades en la duración del sueño y un aumento en pensamientos suicidas en esta población (p.3).

Sigüenza \& Vílchez (2021) observaron, luego de la comparación de muestras pre y post test de ansiedad, que el confinamiento y la presencia de la COVID-19 han provocado un incremento de la ansiedad en los estudiantes, lo cual tiene un papel fundamental en el desempeño académico.

El rendimiento académico está ligado con la salud mental. Serrano, Rojas \& Ruggero (2013) plantean en sus investigaciones que el rendimiento escolar de los estudiantes universitarios guarda relación con estados de depresión y ansiedad, luego, los síntomas depresivos interfieren la actividad intelectual generando el fracaso escolar (p. 57).

Igualmente, Maldonado, Hidalgo y Otero (2000) plantean que un nivel elevado de estrés académico:

Altera el sistema de respuestas del individuo a nivel cognitivo, motor y fisiológico. La alteración en estos tres niveles de respuestas influye de forma negativa en el rendimiento académico y en algunos casos disminuye la calificación de los estudiantes en pruebas orales y escritas. (p. 12) 
Marenco, Suárez, \& Palacio (2017) realizaron un estudio sobre Burnout académico y salud mental en donde se seleccionaron 204 estudiantes de manera intencional-no probabilística, entre los 16 a los 39 años de las Facultades de Ingenierías y Ciencias de la Salud de una universidad pública del caribe colombiano. Los resultados indican que a mayor agotamiento, cinismo e ineficacia se incrementan los síntomas relacionados con problemas de salud mental; del mismo modo, la severidad o nivel del burnout es proporcional a los problemas de salud mental. Se concluye que el agotamiento es relevante en la presencia de síntomas, y el nivel alto de burnout académico es de gran importancia clínica, al coexistir con sintomatología diversa.

\section{METODOLOGÍA}

\section{Objetivo}

Caracterizar los efectos que la pandemia COVID-19 ha generado en la salud mental y el desempeño académico de los y las estudiantes de la Unicamacho.

\section{Tipo de Estudio}

Para el presente estudio se aplicó una metodología mixta, cualitativa y cuantitativa de tipo descriptivo de corte transversal.

\section{Unidad de análisis}

Población de Estudio: estudiantes universitarios Institución Universitaria Antonio José Camacho.

Se realizó una selección por conveniencia para garantizar la facilidad de acceso a las y los estudiantes en tiempos de confinamiento y se obtuvo datos de un total de 60 estudiantes de la Facultad de Ciencias Sociales y Humanas, Facultad de Educación a Distancia e Ingenierías de la Institución universitaria Antonio José Camacho de Cali. Estos estudiantes fueron 41 (67\%) mujeres y 19 (30\%) hombres. De edad entre 19 y 25 años. La participación por facultades fue la siguiente: $8(13 \%)$ estudiantes de Ingenierías, 43 (72\%) estudiantes de Ciencias Sociales Humanas, 2 (3\%) estudiantes de Administración y 7 (12\%) estudiantes de Educación.

\section{Instrumentos}

Se elaboró y aplicó un Instrumento cuantitativo tipo encuesta online, compuesto por 30 preguntas que indagan sobre las afectaciones de la pandemia a nivel emocional, en la vida familiar y cotidiana y en el desempeño académico de las y los estudiantes.

Posteriormente, se aplicó un instrumento cualitativo a los participantes, tipo entrevista semi estructurada para identificar los sentidos y significados que ellos atribuyen a su experiencia educativa en medio de la pandemia. Esta información quedó consignada en audio y diarios de campo realizados por los estudiantes del curso de sistematización.

\section{Procedimiento}

Este ejercicio se realizó con el apoyo de las y los estudiantes de la clase de sexto semestre de Trabajo Social 2020-2 del curso Sistematización, quienes participaron como actores de la experiencia de investigación. Ellos apoyaron el diseño de la investigación y fueron los primeros en responder los instrumentos y fueron ellos quienes establecieron contactos y relación de confianza con otros estudiantes llevándolos a participar. Ellos identificaron los participantes y aplicaron las entrevistas semi estructuradas y apoyaron parte del análisis de la información.

La recopilación de información cuantitativa se hizo por medio de la Herramienta Forms de Microsoft, aplicación de formularios y cuestionarios en línea, lo cual permitió que la información obtenida de los cuestionarios quedase organizada automáticamente en un una base de datos .csv y .txt. Una vez realizada la recopilación y registro de los datos la matriz, estos se sometieron a un proceso de análisis estadístico que nos permitió establecer distintos análisis de los datos obtenidos.

La recopilación de información cualitativa se hizo por medio de grabación de voz de las entrevistas semiestructuradas y por medio de los diarios de campo que realizaron las y los estudiantes. Se hizo la transcripción, lectura y organización de esta 
información en una matriz de doble entrada, donde de manera vertical se ubicaron las personas que respondieron la entrevista y de forma horizontal se pusieron las respuestas al cuestionario según categorías de análisis, de allí surgió una matriz que permitió hacer análisis cruzados de la información. campo que realizaron las y los estudiantes. Se hizo la transcripción, lectura y organización de esta información en una matriz de doble entrada, donde de manera vertical se ubicaron las personas que respondieron la entrevista y de forma horizontal se pusieron las respuestas al cuestionario según categorías de análisis, de allí surgió una matriz que permitió hacer análisis cruzados de la información.

\section{RESULTADOS}

\section{Salud mental de los estudiantes participantes}

A la pregunta: ¿De qué manera considera que la pandemia afectó su cotidianidad?

- "Sí, nos afecta emocionalmente, provocando frustración"

- $\quad$ "Sí, puesto que ya no debía desplazarme a la Universidad, muchas de mis actividades sociales estaban en ella".

- "Sí, porque no puedo interactuar con mis compañeros y maestros. Los dispositivos que hay en mi casa no están en las mejores condiciones para recibir clases".

- "Sí, considero que esto me ha afectado más que todo en mis estudios, porque ya todo no es como antes, estudiar virtualmente no es lo mismo, no se aprende igual, porque estas con mucha presión de que si el Internet falla o la energía se va o tu medio de comunicación se daña; todo lo que puede ser práctico no se puede desarrollar, es cohibirse a muchas cosas y afrontar muchos obstáculos, entonces siempre ha sido difícil adoptarse a estos cambios".
- Por supuesto que afectó la cotidianidad. Afectó de la siguiente manera: primero, ya no se puede asistir a clase presencial; segundo, la carga académica es grande; tercero, tengo hijos que estudian, de igual forma están en confinamiento; cuarto, el trabajo es más limitado; quinto, el solo hecho de estar 24/7 en confinamiento es estresante. Además, también es lidiar con las demás personas, teniendo en cuenta los diferentes temperamentos. Por último, hasta dormir es difícil. Realmente lo cambió todo".

- Sí, de varias maneras. En primer lugar, la manera en que estudiaba, la interacción con docentes y compañeros cambió mi entorno social, económico, por la dificultad de obtener ingresos; político, por la participación en grupos de estudio y en organizaciones, medio ambiental y cultural".

\section{¿CONSIDERA QUE LA PANDEMIA LE HA AFECTADO A NIVEL DE CAMBIOS EMOCIONALES $Y$ DE COMPORTAMIENTOS?}

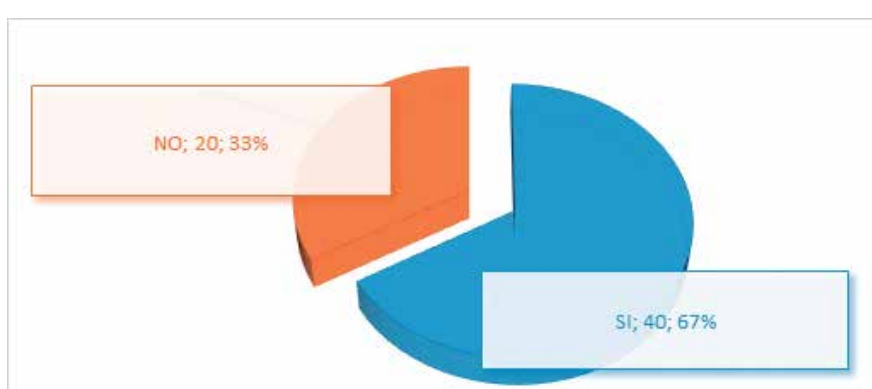

Fig. 1. Cambios emocionales y de comportamiento por la pandemia.

Fuente: Elaboración Propia

\section{¿De qué manera?}

- "Estrés permanente".

- "En mi caso, estar tanto tiempo encerrada me genera estrés y en cuanto al comportamiento a veces no tolero que digan algo".

"Creo a todos nos ha afectado, ya sea porque dejaZmos de hacer lo que nos gustaba o se 
realizaron cambios drásticos como en la manera de trabajar, lo que ha implicado en algún momento agotamiento, pensar que a cada momento nos encontramos aburridos y desesperados por salir o volver a la normalidad".

- "El estar encerrado y con el temor del virus me estresa y hasta me enferma".

- "En cuanto a los cambios emocionales, el aumento de estrés por la manera en que estamos trabajando en la Universidad".

- "Ansiedad, el estar encerrados me produce mucha angustia".

- "Cuando escuchaba las noticias acerca de lo que sucedía, me asusté un poco, me llené de pensamientos de incertidumbre, porque apenas estaba empezando la pandemia y ya estaban diciendo que uno no podría regresar a la vida normal, entonces eso me llenó de pánico y de preocupación, me asusté mucho porque pensé que, si volvíamos a la vida normal muy rápido, estaría propensa a contagiarme del virus y contagiar alguien de mi familia”.

\section{¿HA SUFRIDO EPISODIOS DE ANSIEDAD, CRISIS DE PÁNICO $O$ ANGUSTIA?}

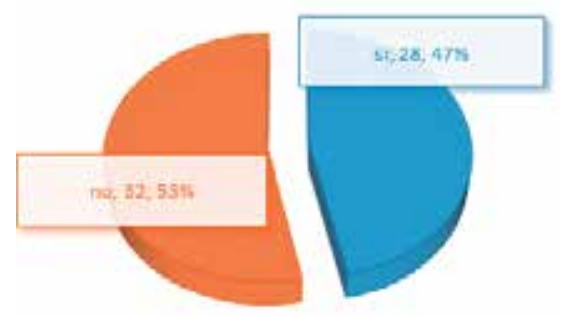

Fig. 2. Episodios de ansiedad, pánico o angustia por la pandemia. Fuente: Elaboración Propia
¿TIENE DIFICULTADES PARA CONCILIAR EL SUEÑO?

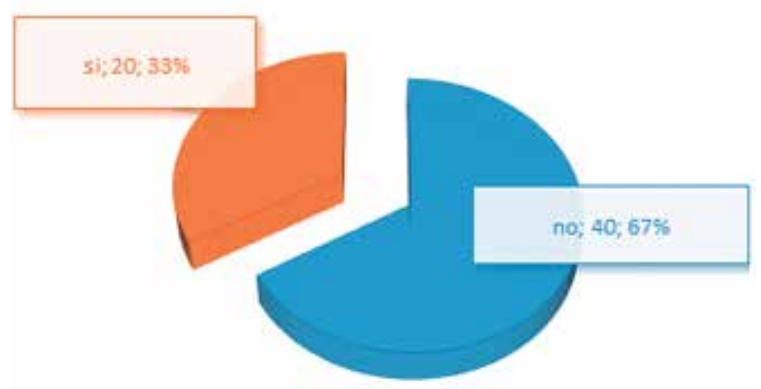

Fig. 3. Dificultades para conciliar el sueño. Fuente: Elaboración Propia

\section{¿SIENTE QUE SU ESTADO DE ÁNIMO ESTÁ CAMBIANDO NEGATIVAMENTE?}

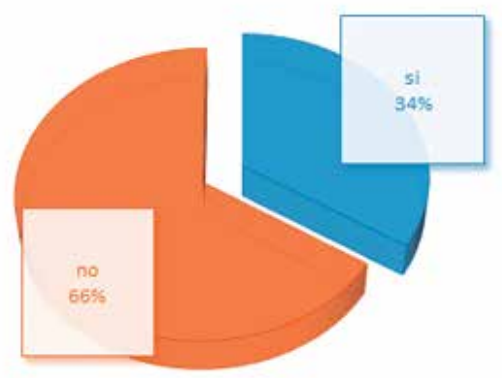

Fig. 4. Estado de ánimo negativo por la pandemia. Fuente: Elaboración Propia

\section{¿CUÁL ES LA EMOCIÓN QUE MÁS PREDOMINA EN USTED EN ESTE ÚLTIMO TIEMPO?}

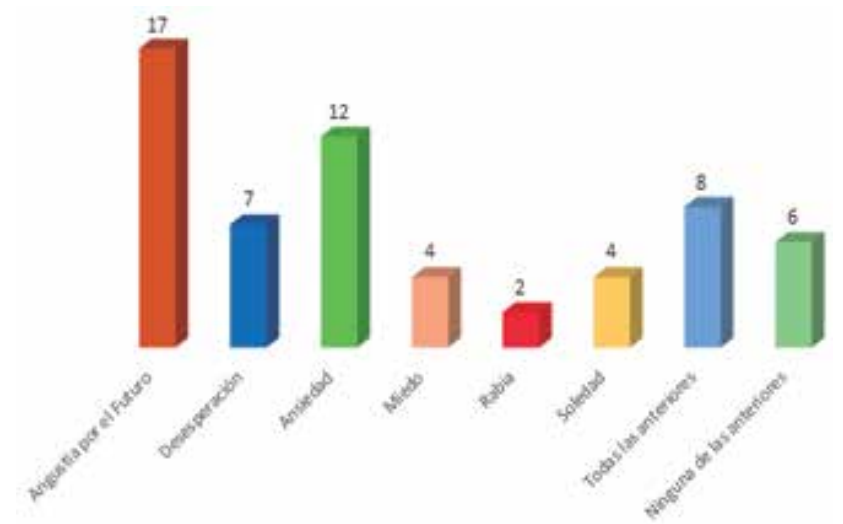

Fig. 5. Emociones que predominan en la pandemia. Fuente: Elaboración Propia 


\section{¿LA PANDEMIA HA TRAÍDO CRISIS ECONÓMICA A SU FAMILIA?}

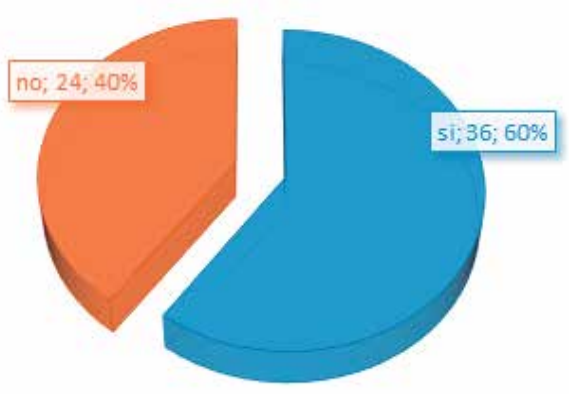

Fig. 6. Afectaciones económicas familiares por la pandemia. Fuente: Elaboración Propia

- “Han habido despidos de empleos de algunos de mis familiares cercanos, por ende, hemos sido obligados a buscar otras maneras de buscar dinero para traer a casa".

- $\quad$ "Mi madre está desempleada, la cual era la proveedora y quien suplía los gastos de la casa”.

- "Porque muchos de los integrantes están desempleados".

\section{¿SE SIENTE AHORA DEMASIADO CANSADA O CON POCA ENERGÍA?}
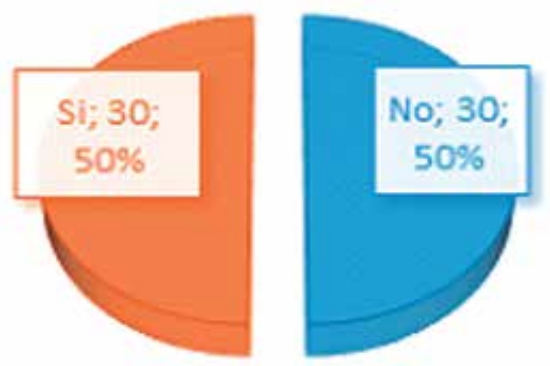

Fig. 7. Efectos en el Desempeño academico por la pandemia. Fuente: Elaboración Propia

\section{¿LE CUESTA INICIAR 0 CULMINAR SUS TAREAS?}

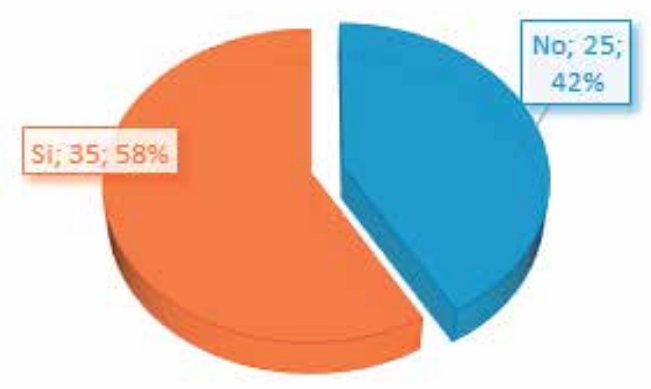

Fig. 8. Efectos en el Desempeño academico. Fuente: Elaboración Propia

\section{¿CONSIDERA QUE SE HA VISTO AFECTADO SU RENDIMIENTO ACADÉMICO POR LA PANDEMIA?}

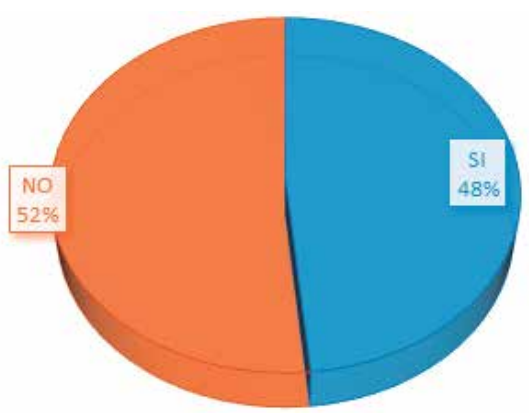

Fig. 9. Efectos en el Desempeño academico. Fuente: Elaboración Propia

\section{¿DE QUÉ MANERA?}

- "Cuando se va el Internet me entra frustración no poder estar presente en la clase, porque perderé conocimientos de la misma”.

- $\quad$ "Por los incidentes que se me presentan a la hora de ingresar a clases, no puedo hacer las cosas de la mejor manera”.

- $\quad$ "Pues me parece un poco más complicado por la cantidad de trabajos, pero en general las clases considero que es más un proceso autónomo que debemos llevar respecto a poner atención en la clase para entender los temas, ya que es más fácil de distraerse". 
- "Lograr concentrarse en la clase o en los temas de las clases es más complicado".

- “Tengo dificultades para concentrarme. me siento la mayoría del tiempo cansada o fatigada”.

- "Algunas veces por fallas del Internet he tenido notas más bajas por el retraso de trabajos".

- $\quad$ "Ya que al comienzo de la pandemia volví a la vereda donde pertenezco y en esta zona no hay señal, y el Internet se cae mucho cuando llueve, no puedo muchas veces entregar los trabajos a tiempo o asistir a clases, entonces por esos motivos me afecta mucho académicamente".

\section{CONCLUSIONES}

Se determinó que efectivamente la salud mental de una buena parte de las y los estudiantes fue fuertemente afectada por la pandemia Covid-19. El encierro y la falta de contacto con experiencias y personas generaron altibajos emocionales, estrés y ansiedad. El cambio de rutinas generó desorientación y frustración. Aumentó la irritabilidad como reacción a partir de sentimientos de ira-enojo que se genera fácilmente. Se encontraron emociones negativas como miedo, incertidumbre, frustración, cansancio y ansiedad intensificada.

Se pudo evidenciar que el rendimiento académico de las y los estudiantes fue fuertemente afectado por la pandemia Covid-19, entre otras razones, por la modalidad virtual, por las dificultades de conexión, por las múltiples entregas de compromisos académicos y las evaluaciones por estos medios. Estos han sido factores altamente estresores en estos tiempos, sobre todo porque gran parte de las y los estudiantes no tienen las condiciones tecnológicas y habitacionales adecuadas, ni métodos de estudio y trabajo en el hogar para dar adecuada respuesta académica.
El traslado de estudiantes a sus regiones y zonas rurales tiene un efecto negativo en la salud mental, pues donde están no tienen condiciones para cumplir con compromisos académicos y eso genera fuertes impactos emocionales.

Con la pandemia las y los estudiantes cumplen desde sus hogares con las responsabilidades académicas y a la par deben asumir y cumplir las obligaciones del hogar (aseo, alimentación, cuidado de otros) y en muchos casos la obligación de generar recursos económicos para el hogar. Estas actividades las realizan sincrónicamente, a las mismas horas que toman las clases. En el hogar les es imposible concentrarse $100 \%$ en el acto educativo, reduciéndose de esta manera la calidad del aprendizaje. Este multitasking obligado del hogar ha aumentado el estrés y la ansiedad a los estudiantes llevando a muchos estudiantes a ese estado de burnout académico que plantean Marenco, Suárez, \& Palacio. (2017).

\section{RECOMENDACIONES}

Se sugiere a la universidad fortalecer el trabajo psicosocial que viene desarrollando para la atención a este fenómeno. Vincular más fuertemente al Trabajo Social para así trabajar más cerca a las personas, acompañando procesos y actuado sobre factores que afectan el desarrollo académico como la familia, la economía, lo socioemocional y las condiciones de acceso y manejo de la virtualidad.

A los docentes se sugiere revisar sus estrategias de trabajo académico y equilibrar, en conjunto, la cantidad de actividades asignadas cuidando no sobre saturar el tiempo de trabajo independiente de las y los estudiantes pues ha sido esto un factor estresor que afectó su rendimiento en 2020.

En temas de atención a la salud mental se sugiere no esperar a que los estudiantes pidan ayuda, porque no suelen hacerlo, mejor hacer equipos de rastreo e identificación de casos con estudiantes en práctica y representantes de grupos. 
Trabajar a nivel de la adaptación al nuevo entorno educativo virtual dadas sus condiciones de acceso, hardware y software de calidad, y conectividad suficiente.

Trabajar a nivel psicosocial en aspectos como:

- $\quad$ Actitud positiva como predisposición mental hacia lo positivo de la vida, así como lo apreciativo, valora "todo" a más.

- Contar con un grupo humano familiar que dé soporte afectivo-emocional y sentimiento de seguridad.

- El deseo de salir adelante con la vida, de progresar y tener éxito. "La necesidad de progresar, estar bien y sentirme bien”.

- Establecimiento de redes de apoyo emocional y académico.

- Hacer trabajo de desarrollo de inteligencia emocional y manejo de situaciones traumáticas.

\section{REFERENCIAS BIBLIOGRÁFICAS}

Capellá, A. (2001). Modelos y paradigmas en salud mental. Revista de la Asociación Española de Neuropsiquiatría, (80), 3-5. http://scielo. isciii.es/scielo.php?script=sci_arttext\&pid=S0 211-57352001000400001\&lng=es\&tlng=es.

Carrazana, V., (2003). El concepto de salud mental en psicología humanista-existencial. Ajayu Órgano de Difusión Científica del Departamento de Psicología UCBSP, 1(1), 1-19. http://www.scielo.org.bo/scielo.php?script= sci_arttext\&pid=S2077-21612003000100001\& $\operatorname{lng}=\mathrm{es} \& \operatorname{tn} g=\mathrm{es}$.

Cobo-Rendón, R., Vega-Valenzuela, A., \& García-Álvarez, D. (2020). Consideraciones institucionales sobre la Salud Mental en estudiantes universitarios durante la pandemia de Covid-19. CienciAméRica, 9(2), 277-284. doi:10.33210/ca.v9i2.322
Contreras, F., Esguerra, G., Haikal, A., Polanía, A., Rodríguez, A., \& Espinosa, J. C.

(2005).

Autoeficacia, ansiedad y rendimiento académico en adolescentes. Diversitas: Perspectivas en Psicología, 1(2), 183-194. http://www.scielo.org.co/scielo.php?script=sci_ arttext\&pid=S1794-99982005000200007\&lng= en\&tlng=es.

Dávila, H. (199). La Salud mental. Difusión en Investigación. Ministerio de Desarrollo Humano - Secretaria Nacional de Salud, 7, 11-15.

De Zubiría Samper, M. (2007). Marco general. En: Sánchez A. Como prevenir la soledad, la depresión y el suicidio en niños y jóvenes, un manual para que sus hijos vivan mejor. Aguilar Altea.

Gatica-Saavedra, M., Vicente, B., \& Rubí, P. (2020). Plan nacional de salud mental. Reflexiones en torno a la implementación del modelo de psiquiatría comunitaria en Chile. Revista médica de Chile, 148(4), 500-505. https://dx.doi.org/10.4067/s0034-98872020000 400500

Maldonado, M. Hidalgo, M. y Otero, M. (2000). Programa de intervención cognitivo-conductual y de técnicas de relajación como método para prevenir la ansiedad y el estrés en alumnos universitarios de Enfermería y mejorar el rendimiento académico. Cuadernos de Medicina Psicosomática, 53, 3-57.

Mebarak, M., \& Castro, A., Salamanca, M.P., \& Quintero, M.F. (2009). Salud mental: un abordaje desde la perspectiva actual de la psicología de la salud. Psicología desde el Caribe, (23), 83-112. https://www.redalyc. org/articulo.oa?id=21311917006 
Palacio Sa, J. E., \& Mart, Y. (2007). Relación del rendimiento académico con la salud mental en jóvenes universitarios. Psicogente, 10(18). Recuperado a partir de http://revistas. unisimon.edu.co/index.php/psicogente/article /view/1556

Quintero, M. A., Pérez, E., \& Correa, S. (2009). La relación entre la autoeficacia y la ansiedad ante las ciencias en estudiantes del nivel medio superior. Revista Internacional de Ciencias Sociales y Humanidades, SOCIOTAM, XIX(2),69-91.

Ramos Pozón, S. (2015). Una propuesta de actualización del modelo bio-psico-social para ser aplicado a la esquizofrenia. Revista de la Asociación Española de Neuropsiquiatría, 35(127), 511-526. https://dx.doi.org/10.4321/ S0211-57352015000300005

Serrano Barquín, C., Rojas García, A., \& Ruggero, C. (2013). Depresión, ansiedad y rendimiento académico en estudiantes universitarios. Revista Intercontinental de Psicología y Educación, 15(1), 47-60. https://www.redalyc.org/articulo.oa? $\mathrm{id}=8$ 0225697004

Sigüenza Campoverde, W., \& Vilchez, J. (2021). Aumento de los niveles de ansiedad en estudiantes universitarios durante la época de pandemia de la COVID-19. Revista Cubana de Medicina Militar, 50(1), e0210931. http://www.revmedmilitar. sld.cu/index.php/mil/article/view/931

\section{AUTORES}

Jimmy Ramírez Bejarano: trabajador Social, Magister en Educación y Desarrollo Humano, Docente, Investigador Institución universitaria Antonio José Camacho. Correo: jimmyaramirez@admon.uniac.edu.co

Ivonne Melisa Lemos Carabalí: estudiante de Trabajo Social Institución universitaria Antonio José Camacho, sexto semestre.Correo: imlemos@estudiante.uniac.edu.co Johana Andrea Moncayo Eraso: estudiante de Trabajo Social Institución universitaria Antonio José Camacho, sexto semestre. Correo: johanamoncayo48@gmail.com

Santiago Martínez Bolaños: estudiante de Trabajo Social, Institución universitaria Antonio José Camacho, sexto semestre. Correo: santiago2018martinez@gmail.com 